\title{
Template Matching Application In Geo-Referencing Of Remote Sensing Temporal Image
}

\author{
Ankit Kumar ${ }^{1}$, Ashish Joshi ${ }^{2}$, Anil Kumar ${ }^{3}$, Ankush Mittal ${ }^{4}$ and D R Gangodkar ${ }^{5}$ \\ ${ }^{1}$ Assistant Professors, Dev Bhoomi Institute of Technology, Dehradun \\ ${ }^{2}$ Assistant Professors, THDC Institute of Hydropower Engineering \& Technology, UK \\ ${ }^{3}$ Scientist, IIRS-ISRO, Dehradun \\ 4, Director Research, Graphic Era University Dehradun \\ ${ }^{5}$ Dean International Affairs, Graphic Era University Dehradun \\ \{mrankitgoyal,a.joshicse1986,dr.ankush.mittal\}@gmail.com \\ \{iirs_anil,dgangodkar\}@yahoo.com
}

\begin{abstract}
This paper focus on template matching approach for tracking object for remote sensing images. Such types of image are source of information which is largely used for information extraction. In this paper, two approaches SAD (sum of absolute differences) and SSD (sum of squared differences), template matching for finding the object. Template matching has provided basics and advance functionality for the image processing, object recognition, features based template matching and many more in a variety of remotely sensed Images. A variety of algorithms exist for comparison of images and making the template matching process fast and reliable. This comparative study with implementation approach focus on the core basics of the template matching in remote sensing images. The contribution of this paper is that, help to find the GCP (ground control point). These GCP can be generated in the form of template and these templates can run on the image and temporal image to find the correct location.
\end{abstract}

Keywords: Template Matching, Panchromatic Images, Correlation, Image Registration, $S S D, S A D$

\section{Introduction}

Many people use the Remote sensing data using in different resource management application. In some application only one data image has used. In some application, temporal image are used. Temporal image are those image which are taken of the same area in different time. For example when the satellite takes the picture of area after this, satellite takes another picture of the same area next few days. So there are so many image of the same area at different time. Such type of image are called temporal image. In temporal image they should be geo registered using common reference image. Geo registrations require large number of GCPs (ground control point). These GCP shave to be transferred from reference image to input image during geo registration process. Generally these GCP are transferred manually. If there is one image then transferred the GCP is very easy. If there are temporal images then it is very difficult the transferred the GCP. To reduce this problem template matching approach has been proposed. This approach helps in semi-automatic mode in temporal image geo referencing. Means of geo referencing is to define its existence in physical space, to find its location in map projection or coordinate systems. These both term finding the relation 
between raster or vector image and coordinates. While working in all temporal image must be geo reference using same reference frame (same coordinate). The proposed approach helps to find the object in temporal image very easily and quickly and also help to monitoring the ship near a harbor.

\section{Template Matching Approach}

Template matching has proven to be a promising technology in the field of image processing for the different applications related to remote sensing, medical, and other related areas. A template based approach provides several application frameworks to known digital image processing concepts for detection of various features in the image portions itself providing the required information from the specific image portions. A large variety of application uses the image registrations to gather information from the physical aspects of the image. A template matching in basic is matching the specific objects of the source image using a template image as shown in the figure 1.General approaches for objection recognition can be classified into two broad categories; area based and feature based approach. Area based approach sometimes called correlation types deal with the images without attempting to detect salient objects window of preferred size or the search window is used for estimation of objects. Whereas feature based methods focus on matching features of the images using contrast, color, hue, saturation etc.

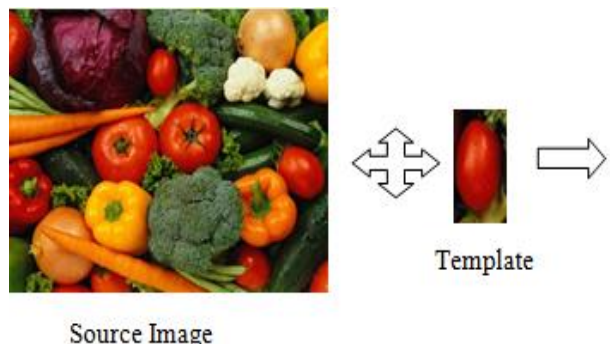

Source Image

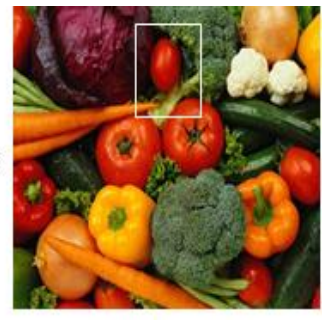

Match Result

Figure 1. Shows the Process of Template Matching

Image registration has also been an important point while the comparison of images or temporal data study [1] showed the relevance on image registration from different sensors and different viewpoints with targets in satellite images, matching stereo images and the basic technology used in the registration process couple of areas included in the paper shows feature based, search space, similarity metric, etc. areas covered in it are the medical remote and computer vision. Similar works are reflected [2] also reflected the transformation measures like Euclidian, similarity affine etc., with registrations methods as pixel based, feature based, contour based, multi modal etc., Similarly [2] showed the recent as well as classic image registration methods with application like in computer vision, methodologies in registration etc.

Template matching is the process of indenting any object in the main image better called as source image with a template, small portion or any different image. It can be achieved through a variety of methods like SAD (Sum of Absolute Difference), NCC (Normalized Cross-Correlation) etc., having different computation measures for processing in the source image and the template image itself. [11] Showed how a basic process of template matching can be enhanced to a time variant scale to make template matching faster and thus making it more suitable to multi scale template matching thus replacing multiple element-by-element floating point multiplications with several additions thus significantly improves the speed. [6] 
In the paper displays a comparative and largely used area based search techniques it provides a view of classic and recent area based methods used. Classifies area based search into further categories as:

1. Cross-correlation based

2. Fourier based

3. Mutual Information based

4. Optimization methods (simulated annealing) etc.

Degree of similarity between methods using vectors between two image $A=(x 1, y 1)$ and $B$ $=(\mathrm{x} 2, \mathrm{y} 2)$ is to be given in the form of dot product given by $\mathrm{AB}=\mathrm{x} 1 \mathrm{x} 2+\mathrm{y} 1 \mathrm{y} 2$ in general images [6]. Coarsening defined in equation (1).

$$
\mathrm{C}_{\mathrm{X}, \mathrm{Y}, \mathrm{D}}=\frac{\sum_{y}^{N} \sum_{x}^{N}\left(L_{x, y} R_{x+D, y}-\mu_{L} \mu_{R}\right)}{\sqrt{\sum_{x}^{N} \sum_{y}^{N}\left(L_{x, y}^{2}-\mu_{L}^{2}\right)} \sqrt{\sum_{x}^{N} \sum_{y}^{N}\left(R_{x+D, y}^{2}-\mu_{R}^{2}\right)}}
$$

Where $\mathrm{X}, \mathrm{Y}$ defines the block location in the source image and $\mathrm{D}$ is the disparity parameter. It computes the "tie points" of the images and the results are been displayed. It is used by robots for exploring its environment as a work proposed [8] through matching sub regions in the image.

Traditional matching algorithms constitutes of the conventional methods that were proven to be computationally intensive and time consuming by recent studies .e.g., of methods include SAD NCC [12]. Which are given as equation (2) and equation (3);

$$
\operatorname{SAD}(\mathrm{x}, \mathrm{y})=\sum_{i=1}^{n} \sum_{j=1}^{n}(T(i, j)-I(x+i, y+j))
$$

Ideally SAD score in this case must be 0 if template is taken from the same image itself but in case if the template is taken from the other registered image of the same area or the other image or the template is itself available these have to calculate the minimum score over the search region of the source image portions [12].In case of NCC which is given as eq 3

$$
\operatorname{NCC}(\mathrm{x}, \mathrm{y})=\frac{\sum_{i=1}^{M} \sum_{j=1}^{N}(I(x+i, y+j) \cdot T(i, j))}{\sqrt{\sum_{i=1}^{M} \sum_{j=1}^{N} I(x+i, y+j)^{2}} \cdot \sqrt{\sum_{i=1}^{M} \sum_{i=1}^{N} T(i, j)^{2}}}
$$

Recent studies on SAD, NCC based matching methods they are comparatively slower as per the requirement. [12] in the paper study showed achieving results faster with multilevel portions using winner update strategy applied in conjunction with an upper bound for cross correlation derived from Cauchy-Schwarz inequality [12] given as shown in eq 4

$$
\sum_{i=1}^{N} a_{i} . b_{i} \leq \sqrt{\sum_{i=1}^{N} a_{i}^{2}} \sqrt{\sum_{i=1}^{N} b_{i}^{2}}
$$

The summation of cross correlation is done into different levels with the partition order determined by the gradient energies of the partitioned regions in the template image itself. Thus, this winner update scheme in conjunction with the upper bound for NCC can be employed to skip unnecessary calculation. [9] Shows the matching process by enhanced bounded correlation that again reduces the number of computation used by 
NCC producing the same results. [5] in the paper showed an matching process using the fourth central movement which forms an estimator in the higher statistics theory, it lowers the impact of the Gaussian noise which may come in the transmission to produce the fast desired results, also uses the BDM (block distortion measure) and SSD (sum of squared differences) [5] which are shown in equations (5) and (6);

$$
\operatorname{BDM}(x, y)=\sum_{l=0}^{n-1} \sum_{k=0}^{m-1} \rho\left(d_{x, y}(k, l)\right)
$$

Where $d_{x, y}=f(x+k, y+l)-g(k, 1), f(x+k, y+l), g(k, l)$ denotes the luminance or other real features

$$
\operatorname{SSD}(\mathrm{x}, \mathrm{y})=\sum_{l=0}^{n-1} \sum_{k=0}^{m-1}\left|d_{x, y}(k, l)\right|^{2}
$$

Considering the various types of the images, our research focus mostly dealing with panchromatic and multispectral images. Paper shows how center of roads can be tracked through least square correlation matching method around a user given input [7]. Shows the use of canny edge detection algorithm for the feature extraction and enhancement of remote sensing images achieving a very high enhancement level [3]. Paper [4] showed the Bayesian way of thinking and introduce a pragmatic approach to extract structural information from RS images by selecting from a library of a priori models those which best explain the structures within an image. Presented Gibbs-Markov random field (GMRF) for the descriptor of the spatial information in remote sensing data [10]. The problem arises in general in these type of images are image is of different bands with different information contained in it like spectral, Radiometric, Textual, Geometric and Contextual etc.

\section{Study Area and Data used}

In our research work image belongs to the one of the famous place of San Francisco Oakland Bridge. This bridge connects the San Francisco and Oakland (figure3). It is used for the passage by the number of vehicles approximately 280,000/day. Beneath the bridge the ocean is present which is used for different types of task by the ships such as passenger ship, tankers, defense, private boats etc. There are different type of harbor and thousands number for ships comes and leave the place. There are so many type of Beach Park where private boats are used by the people for roaming purpose. This image is providing by the world view2. On 8 October 2009 it was launched by Vandenburg Air Force Base in California. It is the first high-resolution satellite with 8-Multispectralimaging bands. Worldview-2 is able to take panchromatic image at $0.46 \mathrm{~m}$ and multiband image at $1.84 \mathrm{~m}$ concurrently. Due to U.S. Government Licensing, the panchromatic imagery will be made available commercially as $0.5 \mathrm{~m}$ imagery. It is able to collect the 975,000 square kilo meters of imagery every day. (376,000 square miles).

\section{Adopted Methodology}

The data used in generic binary consist of an header contains the information about the image such as bands rows columns etc. Whereas the image consists of the digital numbers about which it has to process. We used vector representation for reading and displaying of 
various bands in the tool. The tool was developed in java. The tool functioning consist of various steps in the form first we read the data after reading the rows columns and bands of the image we are displaying it afterwards we have provided a template matching approaches using SAD and the Cross correlation approach SSD for matrix processing. Processing capabilities of our tool are further explained through an algorithmic approach with results showing the implementable mechanism. This methodology adopted has been shown in figure 2 .

\section{Pseudo Code of Template Matching Approach}

Step 0:- Initialize the values in the system. Select the search window as the template image size itself in the Reference image.

Step 1:- Load the panchromatic reference image and the template image.

Step 2:- Compute SAD by moving the template image over the reference image.

Step 3:- SAD will compute to zero as template image has been chosen from the same image itself.

Step 4:-if match is found returns the pixel position where the sad was initially calculates in the reference image.

Step 5:- if no match is found first check the search window has processed the entire reference image if yes return match found. It would be least possible as in our case return not possible

Step 6:- Compute SSD by moving the template image over the reference image

Step 7:-Go to step 4

Step 8:- Again check for the final conditions 


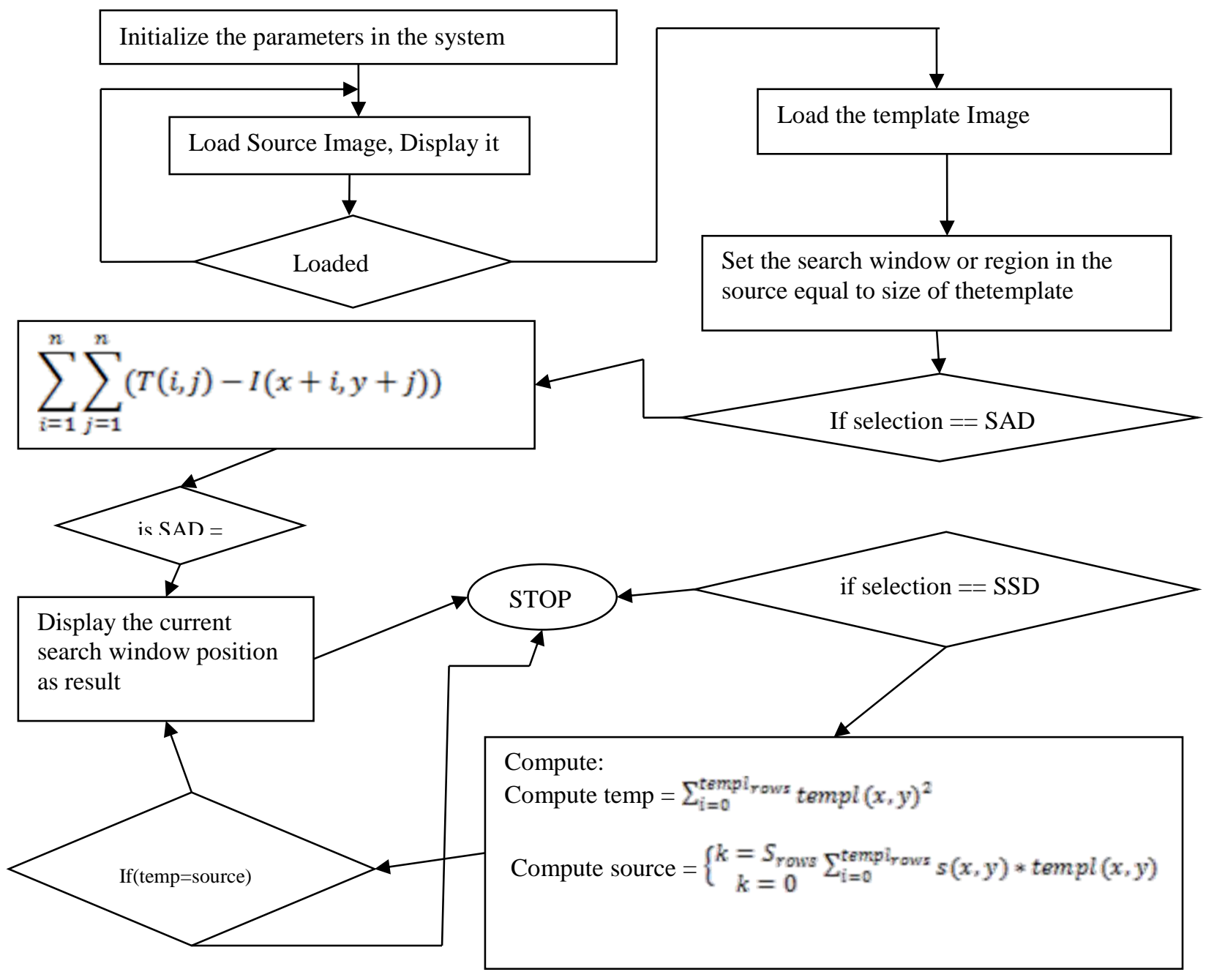

Figure 2. Shows Flow Diagram of Template Matching

\section{Results}

In this application, able to distinguish between the two major templates matching techniques, used for image object analysis. Taken a remotely sensed image for implementation, here if the image is multi band nature it uses as separate vector then apply the template matching approach. This application works like; extract the value of each pixel from the reference image and the template image. Then move the template image on the reference image and find the same pixel value of template and reference image. Because template are taken from the same image. So the pixel value are same. After this where the pixel value is same, where the template image matched. The outcomes show how SAD and SSD mechanisms are implemented besides these the result show that the SSD is computationally intensive for implementation part as the values calculation and then matching leads to more complex mechanisms of implementation. Figure 4 shows the panchromatic reference image, and Figure 5 shows template of the image for research work. 


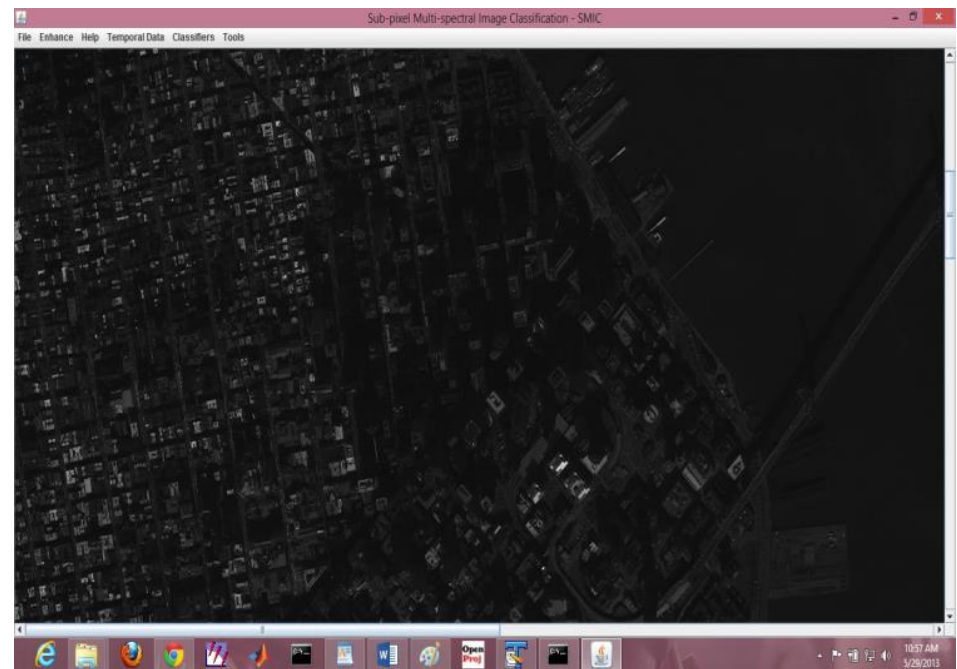

Figure 3. Shows Test Image of San Francisco

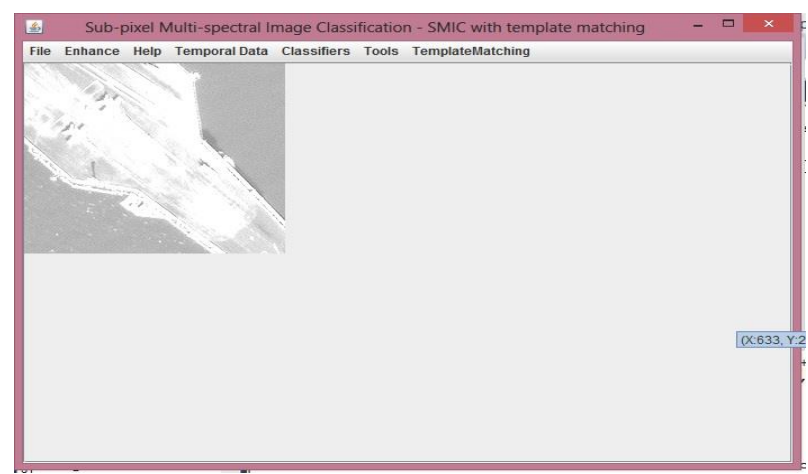

Figure 4. Shows Reference Image

Template images used in this research work, low sizes in the range of $32 \mathrm{X} 32$ and so on the part of it is shown below in Figure 5 The size of the image is very small that's why ,expanded the template size for better understanding the template.

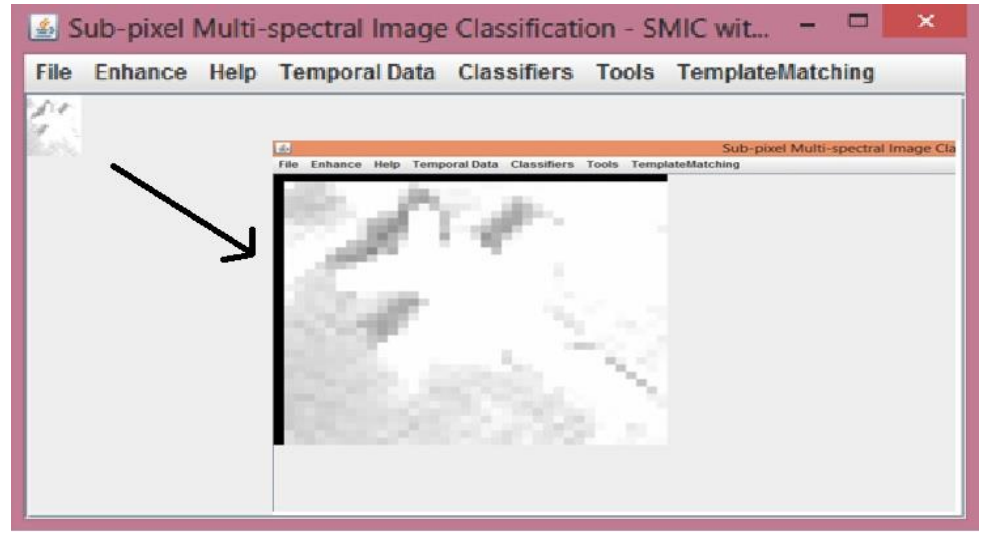

Figure 5. Shows the Template Image and Expanded Template Image 
In Figure 6 show the result of first approach, with SAD approach here template run on the reference image and find the GCP (Ground control point) on the image where the template image match. Here two image are used, first is the reference image and second the template image. The template image move on the reference image top corner of the image and move, one by one column and one by one row on the reference image. If the value matched then find the object on the reference image. Matched result shows in Figure 6. The second approach, which is SSD working, is same like SAD method, result show in Figure 7.

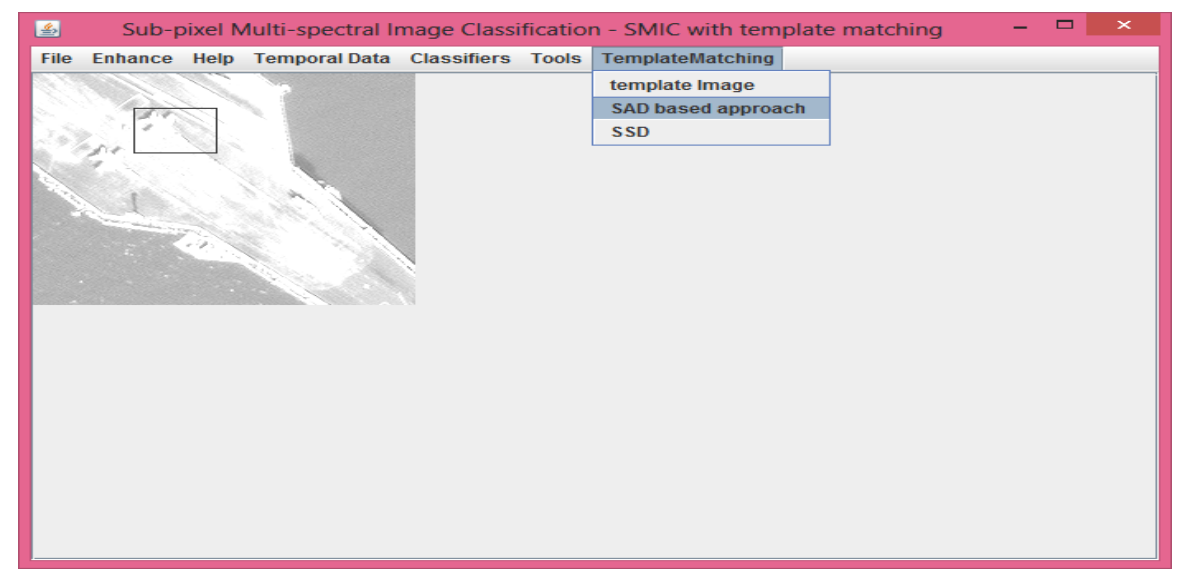

Figure 6. Shows the Match Occurred Using SAD Method

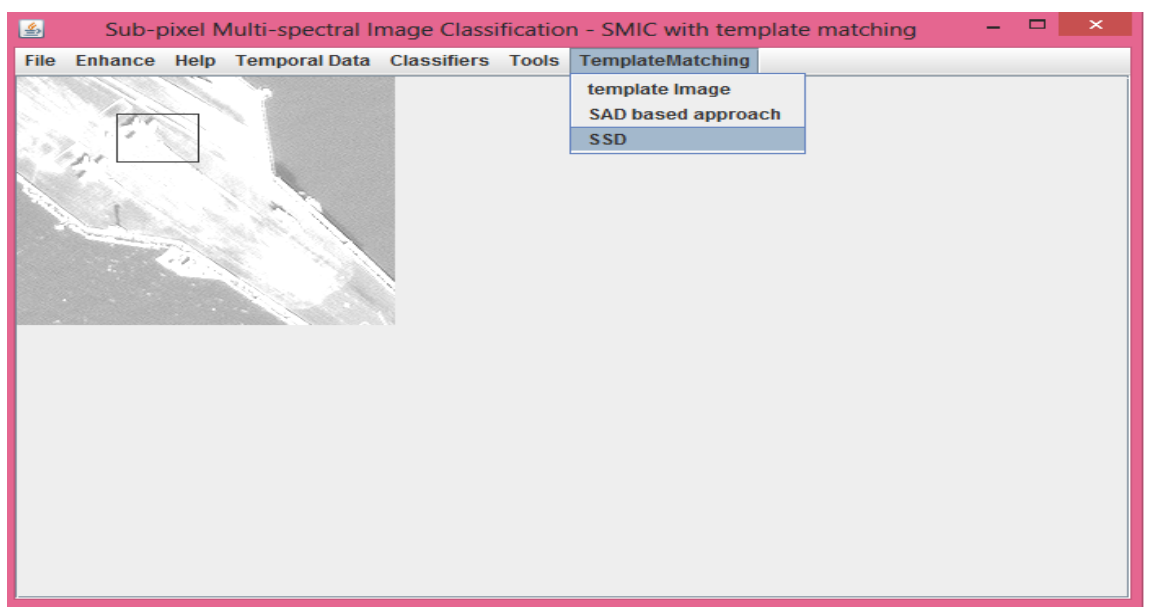

Figure 7. Shows Implementation Results by SSD Method

Experimental results shows that match occurred by both the methods is exact only thing is the time taken by the SAD is more compare to SSD.

\section{Conclusion}

This approach will help to find the GCPs (Ground control point) from the reference image. The (GCPs) can be generated in the form of templates . These templates can run other temporal images on the same area to identify appropriate GCP location. It will help in semiautomatic image registration will identify quickly the GCPs on input image using temporal image of the same area for the image registration. It is much beneficial for semi- automatic 
image registration process where GCP information has to match regardless of the lat-long coordinate not available at the moment. It can be matched with registered image of the same area. Proposed mechanisms found applications like, identifying the moving object in harbors because there are so many ships are moving on the harbors, through this technique ,monitor the which ships is living and enter in the harbor. Through this approach it may be possible to count the object and access the object condition and another application is, recognized the ships which type of ships like defense ships or travel, and also check the activity on the ships. These practical applications may be found suitable for further research.

\section{Acknowledgements}

We are highly thankful to Digital Globe for providing images of worldview-2 the satellite used in our research.

\section{References}

[1] L. G. Brown, "A Survey of Image Registration Techniques”, ACM Computing Surveys, vol. 24, no. 4, (1992).

[2] M. Deshmukh and U. Bhosle, “A Survey Of Image Registration”, International Journal Of Image Processing (IJIP), vol. 5, no. 3, (2011).

[3] M. Ali and D. Clausi', "Using the Canny Edge Detector for Feature Extraction and Enhancement of Remote Sensing Images", Geoscience and Remote Sensing Symposium, IGARSS, IEEE International, vol. 5, (2001), pp. 2298-230.

[4] M. Datcu, K. Seidel and M. Walessa, "Spatial Information Retrieval from Remote-Sensing Images - Part I: Information Theoretical Perspective", IEEE Transactions On Geoscience And Remote Sensing, vol. 36, no. $5,(1998)$.

[5] F. Essannouni and D. Aboutajdine, "Fast Frequency Template Matching Using Higher Order Statistics", IEEE transactions on image processing, vol. 19, no. 3, (2010).

[6] J. Joglekar and S. Gedam, "Area Based Image Matching Methods - A Survey", International Journal of Emerging Technology and Advanced Engineering, vol. 2, no. 1, (2012).

[7] T. Kim, S. Park, M. Kim, S. Jeong and K. Kim, "Tracking Road Centerlines from High Resolution Remote Sensing Images by Least Squares Correlation Matching”, Photogrammetric Engineering \& Remote Sensing, vol. 70, no. 12, (2004), pp. 1417-1422.

[8] M. D. Levine, D. A. O'Handley and G. M. Yagi, "Computer Determination of Depth Maps", Computer Graphic and Images Processing, vol. 2, (1973), pp. 131-150.

[9] S. Mattoccia, F. Tombari and F. Stefano, "Fast Full-Search Equivalent Template Matching by Enhanced Bounded Correlation”, IEEE transactions on image processing, vol. 17, no. 4, (2008).

[10] M. Schroder, H. Rehrauer, K. Seidel and M. Datcu, "Spatial Information Retrieval from Remote-Sensing Images-Part II: Gibbs-Markov Random Fields", IEEE transactions on geoscience and remote sensing, vol. 36 , no. 5 , (1998).

[11] F. Tang and H. Tao, "Fast Multi-scale Template Matching Using Binary Features", IEEE workshop on Applications of Computer Vision (WACV'), (2007).

[12] S. Wei and S. Lai, "Fast Template Matching Based on Normalized CrossCorrelation With Adaptive Multilevel Winner Update”, IEEE Transactions On Image Processing, vol. 17, no. 11, (2008).

[13] B. Zitova and J. Flusser, "Image registration methods: a survey", Image and Vision Computing, vol. 21, (2004), pp 977-1000.

\section{Authors}

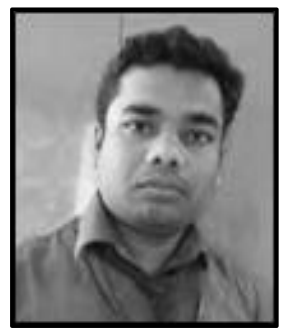

Ankit Kumar, received the MCA, M Tech. Degree Graphic Era University Dehradun, in 2009, 2013.he is currently working with Dev Bhoomi Institute of Technology Dehradun as Assistant Professors. He is Oracle Certified Professional from Oracle University. His areas of interest are Big Data, Soft Computing \& Data Mining, Image preprocessing. 


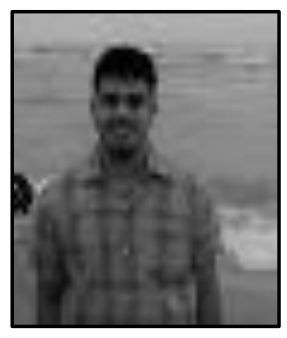

Ashish Joshi, received the M Tech. Degree Graphic Era University Dehradun, in 2013.he is currently working with THDC Institute of Hydropower Engineering \& Technology, UK as Assistant Professors. His areas of interest are Image preprocessing.

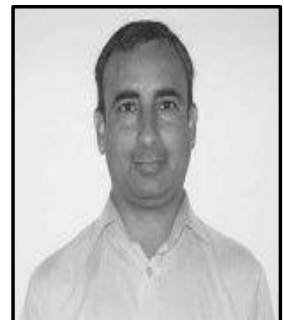

Dr Anil Kumar, received the B Tech, M.E., and Ph.D. Degree in Photogrammetry and Remote Sensing Engineering from IIT Roorkee, in 1992, 1997 and 2007. Presently working in Indian Institute of Remote Sensing (ISRO, Dept. of Space, Govt. of India), Dehradun, India as Scientist/Engineer 'SF'. His areas of interest are Soft Computing application for images, Digital Image Processing, Digital Photogrammetry, LiDAR, and GPS. 